Invited review

\title{
Practical use of azacitidine in higher-risk myelodysplastic syndromes: An expert panel opinion
}

\author{
Pierre Fenaux ${ }^{\mathrm{a}, *}$, David Bowen ${ }^{\mathrm{b}}$, Norbert Gattermann ${ }^{\mathrm{c}}$, Eva Hellström-Lindbergd ${ }^{\mathrm{d}}$, \\ Wolf-Karsten Hofmann ${ }^{\mathrm{e}}$, Michael Pfeilstöcker ${ }^{\mathrm{f}}$, Guillermo Sanz ${ }^{\mathrm{g}}$, Valeria Santini ${ }^{\mathrm{h}}$ \\ a Hôpital Avicenne, Assistance Publique, Hôpitaux de Paris and Paris 13 University, Bobigny, France \\ ${ }^{\mathrm{b}}$ Leeds Teaching Hospital, Leeds, UK \\ ${ }^{\mathrm{c}}$ Heinrich-Heine-Universität, Düsseldorf, Germany \\ ${ }^{\mathrm{d}}$ Karolinska Institute, Huddinge University Hospital, Huddinge, Sweden \\ e University Hospital Mannheim, Berlin, Germany \\ ${ }^{\mathrm{f}}$ Hanusch Hospital, Vienna, Austria \\ ${ }^{g}$ Hospital Universitario La Fe, Valencia, Spain \\ ${ }^{\text {h }}$ Careggi Hospital, Florence, Italy
}

\section{A R T I C L E I N F O}

\section{Article history:}

Received 25 February 2010

Received in revised form 17 May 2010

Accepted 19 May 2010

Available online 6 July 2010

\section{Keywords:}

Myelodysplastic syndromes

MDS

Azacitidine

Recommendations

Int-2

High-risk

\begin{abstract}
A B S T R A C T
Azacitidine is currently the only drug to have shown a significant survival benefit over conventional care regimens in patients with International Prognostic Scoring System (IPSS) intermediate-2 (Int-2) and high-risk myelodysplastic syndromes (MDS), establishing it as an important new treatment for these individuals. However, several aspects of the practical use of azacitidine remain uncertain. This manuscript outlines recommendations discussed by a panel of experts, providing a practical guide for physicians to ensure optimal management of Int-2 and high-risk patients receiving azacitidine.
\end{abstract}

(C) 2010 Elsevier Ltd. All rights reserved.

\section{Contents}

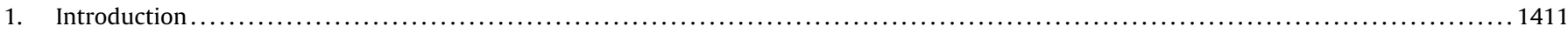

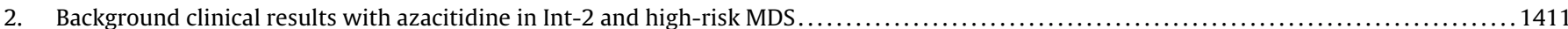

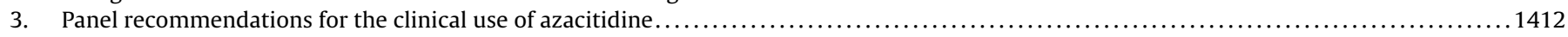

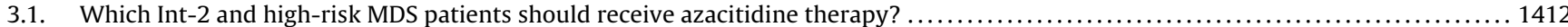

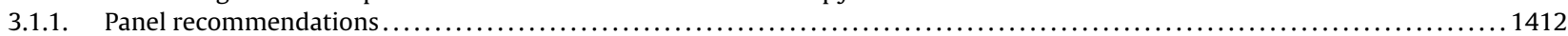

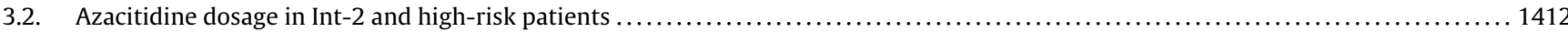

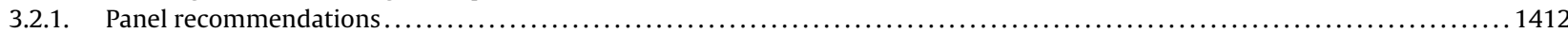

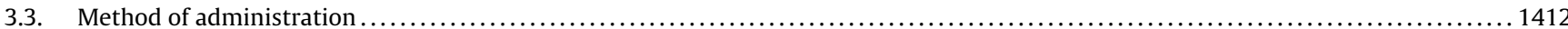

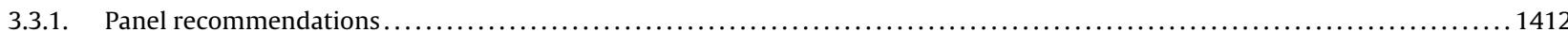

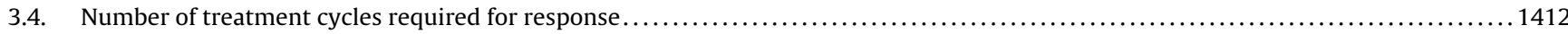

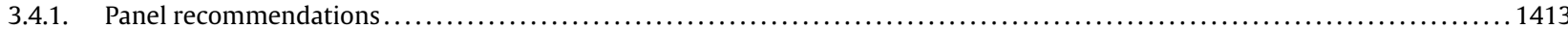

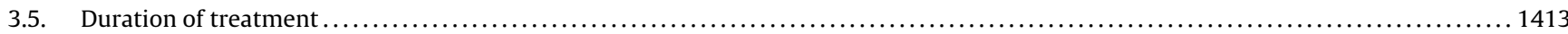

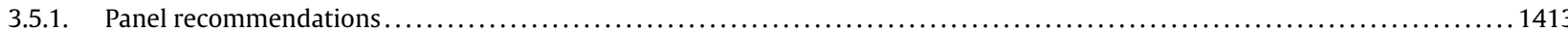

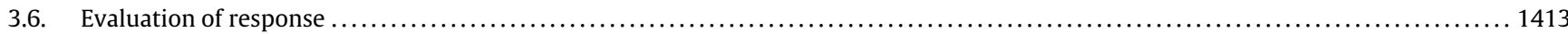

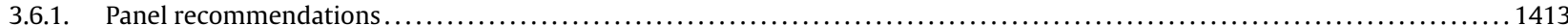

\footnotetext{
* Corresponding author at: Hôpital Avicenne, Assistance Publique, Hôpitaux de Paris and Paris 13 University, 125 rue de Stalingrad, 93009 Bobigny, France. Tel.: +33 148957050; fax: +33 148957058
}

E-mail address: pierre.fenaux@avc.aphp.fr (P. Fenaux). 


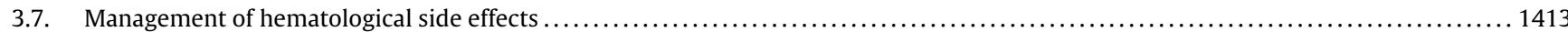

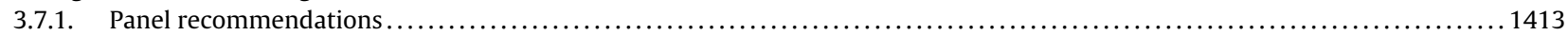

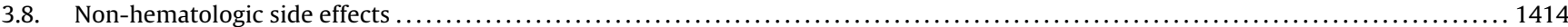

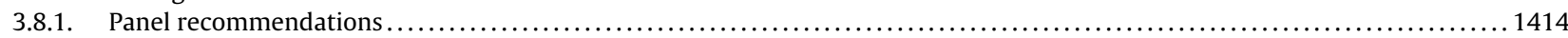

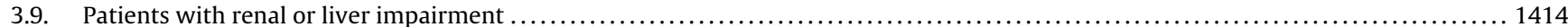

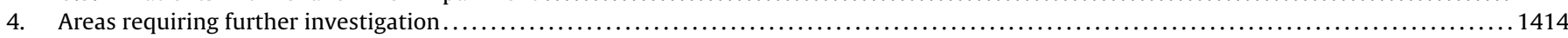

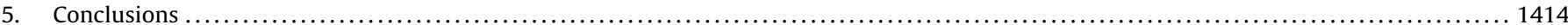

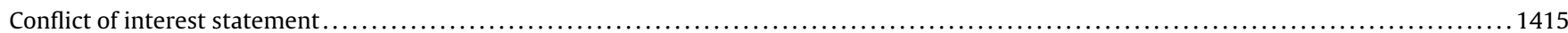

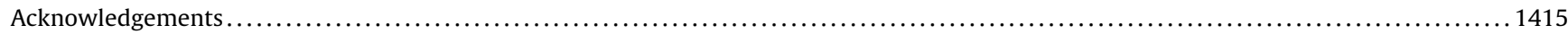

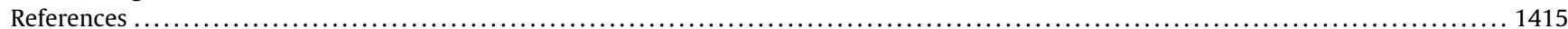

\section{Introduction}

Myelodysplastic syndromes (MDS) are clonal hematopoietic stem cell disorders, predominantly affecting older individuals (median age range from 65 to 75 years) [1] and characterized by ineffective hematopoiesis. This leads to peripheral cytopenias and a substantial risk of progression to acute myeloid leukemia (AML) $[2,3]$.

MDS have been classified morphologically using French-American-British (FAB) criteria [4,5] and more recently by the World Health Organization (WHO) definition [6,7]. Although several prognostic tools exist $[8,9]$, the most widely used is the International Prognostic Scoring System (IPSS), based on the percentage of bone marrow myeloblasts, bone marrow cytogenetics and number of cytopenias [10]. This system stratifies patients into four risk groups: low, intermediate-1 (Int-1), intermediate-2 (Int-2) and high. Patients in the Int-2 and high-risk MDS categories (referred to as higher-risk MDS) have a median overall survival of 1.2 and 0.4 years, respectively and a high risk of progression to AML [10]. These higher-risk patients clearly require treatment capable of modifying the natural history of the disease by prolonging survival.

Allogeneic stem cell transplant (allo-SCT) remains, with few exceptions, the only curative treatment for MDS; however, the vast majority of higher-risk MDS patients are not eligible for allo-SCT due to age, comorbidities and/or the absence of an adequate donor $[4,11]$. Low-dose chemotherapy, such as low-dose cytarabine, has shown no survival advantage over supportive care [12]. Survival rates with intensive chemotherapy are disappointingly low, although a few selected patients such as those $<65$ years with a normal karyotype may achieve prolonged remission [13]. Furthermore, evidence suggests that high-risk patients with unfavorable karyotype anomalies do not benefit from intensive chemotherapy [14].

Improved understanding of the biology of MDS has led to increased interest in novel treatment options, including hypomethylating agents. Silencing of tumor suppressor and other genes that control the cell cycle by hypermethylation appears to play an important role in the pathogenesis and progression of MDS $[15,16]$. Azacitidine (Vidaza ${ }^{\circledR}$, Celgene Europe Ltd., Windsor, UK), a ring analog of cytosine and a deoxyribonucleic acid (DNA) methyltransferase inhibitor (DNMTI) becomes incorporated into both ribonucleic acid (RNA) and DNA [17,18] leading to cytotoxicity to abnormal hematopoietic cells and hypomethylation of DNA [19]. Recently, an international randomized trial conducted in Int-2 and high-risk MDS patients (AZA-001) demonstrated that azacitidine significantly improved survival compared with conventional treatments comprising intensive chemotherapy, low-dose cytarabine and supportive care [20]. The results of this trial led to approval of azacitidine by the European Union for the treatment of adult patients who are not eligible for hematopoietic SCT with Int-2 and high-risk MDS, chronic myelomonocytic leukemia (CMMoL) with $10-29 \%$ blasts without myeloproliferative disorder and AML with $20-30 \%$ blasts (former refractory anemia with excess blasts in transformation [RAEB-T] in the FAB classifica- tion) and multilineage dysplasia, according to the WHO classification [21].

However, because results from AZA-001 study [20] and previous azacitidine studies $[22,23]$ are recent, several aspects of the practical use of azacitidine remain uncertain. Therefore, an international panel of MDS experts who have gathered some experience of azacitidine, were convened to ensure optimal clinical implementation and usage of this novel agent. This paper summarizes the opinions of the group and aims to provide recommendations for the practical use of azacitidine in Int-2 and high-risk patients.

\section{Background clinical results with azacitidine in Int-2 and high-risk MDS}

Clinical results with azacitidine in Int-2 and high-risk MDS are mainly based on two randomized phase III multicenter trials; the CALGB 9221 [22] and AZA-001 [20] studies. In the CALGB 9221 study, azacitidine $\left(75 \mathrm{mg} / \mathrm{m}^{2} /\right.$ day for 7 consecutive days, every 28 days) was compared with best supportive care (BSC) in 191 FABdefined MDS patients (46\% defined as Int-2 or high-risk), with a median age of 68 years [22]. Sixty percent of the azacitidine group, compared with $5 \%$ of control arm patients, responded to treatment according to CALGB response criteria $(p<0.0001)$, with a median time to leukemic transformation or death of 21 months in azacitidine patients versus 12 months in the BSC $\operatorname{arm}(p=0.007)$. No significant difference in survival was observed, possibly due to the crossover design of the study. A significant survival advantage for those patients who initially received azacitidine or who had switched from BSC to azacitidine within 6 months of inclusion in the study was reported in the 6-month landmark analysis $(p=0.03$ ). Furthermore, when the analysis was restricted to patients $\geq 65$ years of age with refractory anemia with excess blasts (RAEB) or RAEB-T, patients treated with azacitidine had a longer median survival than those receiving BSC in spite of the crossover (azacitidine: 19.5 months; BSC: 14 months; $p<0.04$ ) [24].

Further analysis of CALGB 9221 [22] demonstrated delayed onset of red blood cell (RBC; $p=0.007$ ) and of platelet transfusions [24] and significant improvement in quality of life (QoL) for patients receiving azacitidine compared with patients on supportive care [25].

In AZA-001 study, investigators determined which of three conventional care regimens (CCR: intensive AML-like chemotherapy, low-dose cytarabine and BSC) was most appropriate for each of 358 MDS patients prior to randomization. Patients (Int-2 and highrisk patients: $87 \%$; median age: 69 years) were then randomized to azacitidine $\left(75 \mathrm{mg} / \mathrm{m}^{2} /\right.$ day for 7 consecutive days, every 28 days) or CCR [20]. Azacitidine significantly improved median survival (azacitidine: 24.5 months CCR: 15.0 months; $p=0.0001$ ) with a two-fold difference in overall survival at 2 years compared with CCR (azacitidine: 50.8\%; CCR: 26.2\%; $p<0.0001$ ) [20]. Progression to AML was delayed, whilst RBC transfusion requirements $(p<0.0001)$ and rate of infections ( $p=0.0032)$ were also significantly improved with azacitidine. The survival advantage observed with azacitidine 
was seen irrespective of age (including patients aged $>75$ years) [26], percentage of marrow blasts (including patients with $20-30 \%$ blasts, now classified as AML using WHO criteria) [27] or karyotype [20], and was significant compared with BSC and low-dose cytarabine. In this clinical trial, there was no significant difference in overall survival between patients treated with AML-like chemotherapy and those receiving azacitidine; this is likely to be due to the small number of patients in this analysis [20].

\section{Panel recommendations for the clinical use of azacitidine}

\subsection{Which Int-2 and high-risk MDS patients should receive azacitidine therapy?}

The AZA-001 study has shown a significant overall survival advantage with azacitidine over low-dose cytarabine and supportive care in Int-2 and high-risk MDS patients irrespective of age, FAB/WHO classification and karyotype [20]. In addition, the survival benefit was observed in subgroups with the poorest prognosis, including patients with the $-7 /$ del7q cytogenetic abnormality [20].

\subsubsection{Panel recommendations}

- All MDS patients should be classified according to IPSS to determine eligibility for treatment.

- When karyotyping is not available or after technical failure to obtain the karyotype, clinicians are advised to consider patients with a bone marrow blast percentage $>10 \%$ as being at least in the Int-2 IPSS risk category; these patients can therefore be considered as candidates for azacitidine therapy.

- Azacitidine therapy should be considered as the first-line treatment, instead of low-dose cytarabine or BSC, for the majority of Int-2 and high-risk patients ineligible for SCT irrespective of age, karyotype, FAB or WHO classification or concomitant comorbidities. However, there is not yet sufficient evidence to choose between azacitidine and intensive chemotherapy for patients ineligible for SCT but potential candidates for aggressive chemotherapy.

- Most experts agree that intensive chemotherapy is associated with relatively high complete response (CR) rates in patients with a normal karyotype (i.e. an indicator of good prognosis), although the duration of those responses generally does not exceed 12-15 months.

- Patients with an unfavorable karyotype (who typically have a poor response to intensive chemotherapy) responded favorably to azacitidine in the AZA-001 trial; these individuals may therefore be recommended for first-line treatment with azacitidine in the future.

- Accumulated experience has shown that many patients $>75$ years benefit from azacitidine especially because the associated myelosuppression is lower than with treatment options such as low-dose cytarabine (Ara C) chemotherapy. Therefore, only very frail patients may a priori be considered as candidates for supportive care only.

- In rare cases of Int-2 or high-risk MDS with no or limited cytopenias, it is currently unknown whether azacitidine should be started immediately after diagnosis or delayed until more severe cytopenias are apparent or the patient becomes symptomatic.

\subsection{Azacitidine dosage in Int-2 and high-risk patients}

Azacitidine was administered subcutaneously at a dose of $75 \mathrm{mg} / \mathrm{m}^{2} /$ day for 7 consecutive days, every 28 days in both phase III trials published to date; this is currently the only dosing schedule that has shown a survival advantage in Int-2/high-risk patients
[20,22]. Recently, a randomized, multicenter open-label phase Il study ( $n=151$ ) compared three schedules of azacitidine (administered every 4 weeks): a 5-2-2 regimen $\left(75 \mathrm{mg} / \mathrm{m}^{2} /\right.$ day during 5 days, followed by 2 days no treatment (for the weekend) then two other treatment days); a 5-2-5 regimen, with lower daily doses of azacitidine $\left(50 \mathrm{mg} / \mathrm{m}^{2} /\right.$ day for 5 days, followed by 2 days no treatment then another 5 days at $50 \mathrm{mg} / \mathrm{m}^{2} /$ day); or a 5 regimen (azacitidine $75 \mathrm{mg} / \mathrm{m}^{2} /$ day for 5 days) [28]. The three schedules yielded similar response rates, apparently close to that of the approved 7-day dosing schedule. However, in this study, 63\% of patients were diagnosed with lower-risk MDS and the standard dose of $75 \mathrm{mg} / \mathrm{m}^{2} /$ day for 7 consecutive days, every 28 days, was not included. Furthermore, the study was not designed to evaluate overall survival, progression-free survival or rate of evolution to AML [28]. Therefore, there is not yet sufficient evidence to recommend those dosing regimens in patients with Int-2 or high-risk MDS. However, the 5-2-2 regimen is frequently used in institutions where no injections are possible during the weekend.

\subsubsection{Panel recommendations}

- $75 \mathrm{mg} / \mathrm{m}^{2} /$ day azacitidine administered subcutaneously for 7 consecutive days, every 28 days should be the preferred dosing scheme for all Int- 2 and high-risk MDS patients, irrespective of the presence of severe cytopenias at baseline.

\subsection{Method of administration}

One of the first clinical studies with azacitidine demonstrated that a continuous intravenous infusion of $75 \mathrm{mg} / \mathrm{m}^{2} /$ day for 7 days, every 28 days was effective in the treatment of MDS (RAEB and RAEB-T) [29]. However, the majority of subsequent studies demonstrating the clinical efficacy of azacitidine, including both phase III studies, have used a subcutaneous injection of $75 \mathrm{mg} / \mathrm{m}^{2} /$ day for 7 consecutive days every 28 days [20,22].

\subsubsection{Panel recommendations}

- Subcutaneous injection of $75 \mathrm{mg} / \mathrm{m}^{2} /$ day for 7 consecutive days, every 28 days. In the rare cases where local severe side effects persist in spite of adequate prophylactic measures (see Section 3.8.1), the intravenous route may be preferred. In a US multicenter, community-based registry of patients with MDS treated with azacitidine, the intravenous route appeared as efficacious as the subcutaneous route, although this was not a randomized study [30].

- Preliminary results from phase I studies have indicated that an oral form of azacitidine is active and well tolerated with a manageable side effect profile in subjects with MDS or AML, suggesting that this method of administration may be envisaged in the future [31].

\subsection{Number of treatment cycles required for response}

In contrast to standard chemotherapy treatment, several cycles of azacitidine are usually required before a clinical benefit becomes evident $[20,22,23,29]$. For example, in CALGB 9221 trial, $75 \%$ of responders had achieved a response by cycle four with $90 \%$ of the responses observed by cycle six [23]. In AZA-001 study, $81 \%$ of responders achieved a first response by cycle six and $90 \%$ achieved a first response by cycle nine [32]. This apparent delay in response is possibly due to the mode of action of azacitidine which is timedependent on the cell cycle. As azacitidine is incorporated into DNA, it impedes the activity of DNA methyltransferase enzymes and promotes DNA hypomethylation. These actions of azacitidine are S-phase dependent and two or more cell cycles with subsequent 
DNA synthesis appear necessary to reinitiate gene transcription and expression [22].

\subsubsection{Panel recommendations}

- Patients should receive at least six cycles of azacitidine before any treatment decisions are made as to continuing therapy, unless frank progression is observed before completion of those six cycles.

\subsection{Duration of treatment}

In the AZA-001 study, patients received a median number of nine cycles of azacitidine, and responders a median of 14 cycles [32]. This prolonged treatment duration could have contributed to the observed survival benefit [20]. In addition, continued azacitidine dosing after a first response led to a higher IWG response category in $43 \%$ of patients (after approximately four additional treatment cycles) [32]. An earlier study by Silverman et al. suggested that 18 months or more of treatment may be required for the maximal response in some patients [23]. These studies support the need for sufficiently prolonged azacitidine therapy.

A significant survival benefit with azacitidine in the AZA-001 study was demonstrated not only in patients achieving a CR or partial response (PR) but also hematologic improvement (HI) according to IWG 2000 response criteria, mainly corresponding to an erythroid response with RBC transfusion independence or less often platelet response [33]. It is not yet known whether patients treated with azacitidine achieving stable disease without HI have an overall survival benefit.

\subsubsection{Panel recommendations}

- Maintain azacitidine treatment in patients achieving CR, PR or HI. - In the absence of studies precisely determining the optimal duration of maintenance therapy, it is recommended to treat those patients until disease progression.

- This recommendation may be especially important in the presence of poor prognostic factors such as monosomy 7, complex karyotype, substantial excess of marrow blasts or severe cytopenias.

\subsection{Evaluation of response}

As discussed, several treatment cycles may be required for the clinical benefits of azacitidine to become evident [22], and a survival benefit is apparent not only in patients who achieve CR and PR, but also in those who achieve HI (i.e. improvement of cytopenias without reduction of bone marrow blasts), despite the persistence of cytogenetic abnormalities. Therefore, unless progression is suspected, evaluating bone marrow aspirates for response to treatment should most likely be delayed until after four or six cycles of treatment. Methylation status may also be discussed when evaluating patients treated with azacitidine. However, consensus on the required techniques to assess DNA methylation and on the genes that should be analyzed is currently lacking owing to its largely experimental nature and lack of clinical validation.

\subsubsection{Panel recommendations}

- Complete blood counts should be performed as needed to monitor response and toxicity.

- Morphological and cytogenetic analysis of bone marrow aspirates is recommended to evaluate response after four to six cycles of azacitidine treatment or earlier if disease progression is suspected.
- If bone marrow aspirates are not evaluable due to hypocellularity or fibrosis, a biopsy will be required after six cycles.

\subsection{Management of hematological side effects}

In the AZA 001 trial, hematologic adverse events (AEs), including neutropenia (azacitidine: 91\%; CCR: 76\%) and thrombocytopenia (azacitidine: 85\%; CCR: 80\%) were the most common AEs reported, although $<5 \%$ of patients receiving azacitidine required treatment discontinuation [20]. During the first 1-2 treatment cycles, cytopenias may become apparent or those present at diagnosis might be exacerbated [28] highlighting the importance of monitoring. Anemia, neutropenia and thrombocytopenia are generally observed during cycles one and two and infrequently thereafter [20]. During the initial cycles of treatment, azacitidine is also associated with increased infection rates [34]; this increase does not appear to adversely affect response or survival which again highlights the importance of monitoring patients receiving azacitidine for AEs [20]. It is, therefore, important for hematologists to advise patients that their symptoms may worsen initially whilst emphasizing the importance of maintaining their treatment.

Management of cytopenias should follow standard guidelines for RBC and platelet transfusions. Therefore, the threshold hemoglobin levels and platelet counts for RBC and platelet transfusions should be similar to those recommended for patients treated with chemotherapy whilst taking account of age and comorbidities. In elderly patients and patients with comorbidities, those infections may be particularly severe. In case of infections, broad spectrum antibiotics are indicated in case of fever. However, there are no established indications for primary or secondary prophylactic quinolone antibiotics or antifungals or for the use of granulocyte colony-stimulating factor (G-CSF). Evaluation of the role of antibiotics and antifungals as prophylaxis in this context is therefore important. The use of G-CSF should probably be of concern only in patients with a high baseline marrow count due to the possible increase of blasts with this agent. Furthermore, the efficacy of growth factors in improving neutropenia has not been demonstrated in high-risk MDS patients receiving low-dose chemotherapy [35]. Further evaluation of G-CSF in treating severe cytopenias in patients with MDS is required and, therefore, G-CSF should probably not be used in the absence of severe infections. Iron chelation therapy is not generally recommended in high-risk patients but may be considered in individuals who respond to treatment and are expected to have relatively prolonged survival as well as in candidates for allogeneic SCT following response to azacitidine treatment. There is currently insufficient data to recommend the use of erythropoietin in combination with azacitidine.

\subsubsection{Panel recommendations}

- Monitoring hematologic AEs.

- Regular monitoring of blood cell counts.

- The risk of aggravated leukopenia and thrombocytopenia requires blood cell counts to be performed weekly in the first three cycles, or more regularly if clinically indicated (e.g. fever). - Subsequently blood cell counts can be assessed every 2 weeks.

- Management of hematologic AEs.

- Dose modifications are defined in the drug's Summary of Product Characteristics according to the extent of baseline cytopenias and their relative deterioration with treatment (Table 1).

- Reducing the dose of azacitidine in each cycle or delaying cycles may be associated with lower efficacy of this agent. Therefore, for patients with advanced MDS (severe pancytopenia, marrow blasts $>15-20 \%$, complex karyotype) dose modifications are not recommended during the first three treatment cycles even in 
Table 1a

Recommended dose modifications in patients without reduced baseline blood counts $\left(\right.$ WBC $>3.0 \times 10^{9} /$ L or ANC $>1.5 \times 10^{9} /$ or platelets $>75.0 \times 10^{9} / \mathrm{L}$ ) prior to first treatment and experiencing hematological toxicity following azacitidine treatment. Following dose modifications, the cycle duration should return to 28 days.

\begin{tabular}{lcc}
\hline Nadir counts & & $\begin{array}{c}\text { \% dose in next cycle if recovery } \\
\text { is not achieved within 14 days }\end{array}$ \\
\hline ANC & Platelet & \\
\hline$\leq 1 \times 10^{9} / \mathrm{L}$ & $<50 \times 10^{9} / \mathrm{L}$ & $50 \%$ \\
$>1 \times 10^{9} / \mathrm{L}$ & $>50 \times 10^{9} / \mathrm{L}$ & $100 \%$ \\
\hline
\end{tabular}

a Recovery $=$ counts $>$ Nadir count $+(0.5 \times$ [baseline count - Nadir count $])$

\section{Table 1b}

: Recommended dose reductions in patients with reduced baseline blood counts $\left(\mathrm{WBC}<3.0 \times 10^{9} / \mathrm{L}\right.$ or ANC $<1.5 \times 10^{9} /$ L or platelets $\left.<75.0 \times 10^{9} / \mathrm{L}\right)$ prior to treatment and experiencing hematological toxicity following azacitidine treatment. Following dose modifications, the cycle duration should return to 28 days.

\begin{tabular}{lll}
\hline Bone marrow cellularity & $\begin{array}{l}\text { \% dose in next cycle if recovery } \\
\text { is not achieved within } 14 \text { days }\end{array}$ \\
\hline & Recovery $<21$ days & Recovery $>21$ days \\
$15-50 \%$ & $100 \%$ & $50 \%$ \\
$<15 \%$ & $100 \%$ & $33 \%$ \\
\hline
\end{tabular}

WBC, white blood cell; ANC, absolute neutrophil count.

a Recovery $=$ counts $>$ Nadir count $+(0.5 \times$ [baseline count - Nadir count $])$.

the presence of severe cytopenias, except in the presence of lifethreatening complications such as sepsis.

- Treatment delays (as defined in Table 1 ) are probably preferable to dose reductions, although they should also be avoided as much as possible, especially during the first three cycles.

- Platelet and RBC transfusions in accordance with standard guidelines for the first few months of therapy to treat cytopenias.

- G-CSF following azacitidine could be considered for patients developing severe neutropenic fever, or as secondary prophylaxis after a severe infectious episode, although evidence for prophylactic use is currently lacking.

- Quinolone antibiotics and/or antifungals could be considered as secondary prophylaxis in patients following an infectious episode. A possible role for primary prophylaxis in patients with prolonged absolute neutrophil count (ANC) of $<500 \times 10^{6} /$ L and predisposition to infection, such as individuals with chronic obstructive pulmonary disease (COPD), would require more thorough evaluation.

\subsection{Non-hematologic side effects}

The most common non-hematologic AEs associated with azacitidine treatment in the AZA-001 study included gastrointestinal and injection site reactions [36]. Of note, the majority of AEs were transient and resolved during the study [36].

\subsubsection{Panel recommendations}

- Nausea and vomiting.

o Oral potent anti 5-hydroxytryptamine type 3 receptor (5-HT3) antiemetics are recommended before each dose of azacitidine.

- Laxatives may be required if constipation occurs following use of antiemetics.

- Injection site reactions.

- Do not purge the air from the syringe prior to injection.

- Gently massage the injected region after the injection has been delivered.

- Ensure injection sites are at least $2 \mathrm{~cm}$ apart.

- Volume per injection site should not exceed $4 \mathrm{~mL}$.

- Ensure injections are not administered to sites which have had a previous reaction.
- In cases where local reactions persist, a local non-steroidal antiinflammatory drug cream or lotion may be applied. A recent report has suggested skin reactions associated with azacitidine may be reduced with use of evening primrose oil lotion, although this needs to be confirmed in further controlled studies [37].

- Intravenous infusion could be considered in the rare patients with severe injection site reactions following subcutaneous administration of azacitidine, despite precautionary measures being taken.

\subsection{Patients with renal or liver impairment}

Several dialyzed patients have been successfully treated with no major cytopenias by reducing the azacitidine dose by about onethird. However, as there is limited experience of treating patients with renal or hepatic impairment, a more systematic evaluation would be necessary to make specific recommendations.

\section{Areas requiring further investigation}

Azacitidine is currently the only agent proven to increase survival in patients with Int-2/high-risk MDS, and it is effective in several difficult-to-manage groups, including the elderly and patients with comorbidities [20]. Further studies with azacitidine are currently underway, including its use:

- Prior to allogeneic SCT in order to reduce tumor burden without excessive toxicity, or to prevent relapse after allogeneic SCT [38].

- As maintenance treatment after intensive chemotherapy $[39,40]$.

- In combination with different agents including histone deacetylase (HDAC) inhibitors, lenalidomide and low-dose chemotherapy $[41,42]$.

- In low/Int-1 risk MDS that are resistant or not suitable for other treatments [43].

- In more 'proliferative' AML, i.e. with >30\% marrow blasts.

\section{Conclusions}

Azacitidine is an important new management approach for individuals with Int-2/high-risk MDS due to its beneficial effects on survival and QoL measures [20,25,34]. However, as results from the pivotal studies with azacitidine are recent, several aspects of the practical use of this agent are still uncertain. The goal of the recommendations presented here is to ensure optimal management of Int- 2 and high-risk patients to maximize clinical responses. Patients should be classified according to IPSS to ensure all Int-2 and high-risk patients ineligible for SCT are considered for azacitidine therapy, irrespective of age, concomitant comorbidities, karyotype, FAB or WHO classification. Based on the evidence currently available, patients should receive azacitidine at a dose of $75 \mathrm{mg} / \mathrm{m}^{2} /$ day for 7 consecutive days, every 28 days for a minimum of six cycles, with maintenance treatment until disease progression in patients achieving CR, PR or HI. Neutropenia and thrombocytopenia are the most common AEs during azacitidine therapy; at least in the most advanced cases of MDS, no dose modifications are recommended during the first three treatment cycles even in the presence of severe cytopenias, except in case of clinical complications (especially sepsis). Management strategies for cytopenias include RBC and platelet transfusions; the prophylactic use of antibiotics, antifungals and G-CSF needs further evaluation. Non-hematologic side effects are generally manageable and short-lived.

Preliminary findings on the efficacy and safety of combinations of azacitidine and other drugs, such as HDAC inhibitors and lenalidomide, are encouraging $[38,40,42]$ but will have to be validated in phase III clinical trials with survival as primary endpoint. 
Indeed, azacitidine is associated with improved survival in spite of modest CR and PR rates; therefore, overall survival rather than response should be the primary endpoint of future studies.

\section{Conflict of interest statement}

Pierre Fenaux and David Bowen have received honoraria from Celgene for advisory boards and invited lectures. Norbert Gatterman has received research support and lecture honoraria from Novartis and Celgene. Eva Hellström-Lindberg has received honoraria for advisory boards and a research grant from Celgene. Wolf-Karsten Hofmann serves as a consultant and has received research support from Celgene. Michael Pfeilstöcker has received speaker honoraria from Celgene. Guillermo Sanz serves as a consultant for Celgene. Valeria Santini serves as a consultant, has received honoraria for advisory boards and received research funding from Celgene.

\section{Acknowledgements}

Research support was provided by Celgene. Editorial/writing support was provided by Dr Marion James, ScopeMedical Ltd., funded by Celgene.

Contributions: The authors were fully responsible for content and editorial decisions for this manuscript.

\section{References}

[1] Phekoo KJ, Richards MA, Moller H, Schey SA. The incidence and outcome of myeloid malignancies in 2,112 adult patients in southeast England. Haematologica 2006;91(10):1400-4.

[2] Cataldo VD, Cortes J, Quintas-Cardama A. Azacitidine for the treatment of myelodysplastic syndrome. Expert Rev Anticancer Ther 2009;9(7): 875-84.

[3] Itzykson R, Fenaux P. Optimal sequencing of treatments for patients with myelodysplastic syndromes. Curr Opin Hematol 2009;16(2):77-83.

[4] NCCN Clinical Practice Guidelines in Oncology ${ }^{\mathrm{TM}}$ : Myelodysplastic Syndromes [computer program]. Version 22010.

[5] Bennett JM, Catovsky D, Daniel MT, Flandrin G, Galton DA, Gralnick HR, et al. Proposals for the classification of the myelodysplastic syndromes. BrJ Haematol 1982;51(2):189-99.

[6] Vardiman JW, Harris NL, Brunning RD. The World Health Organization (WHO) classification of the myeloid neoplasms. Blood 2002;100(7):2292-302.

[7] Vardiman JW, Thiele J, Arber DA, Brunning RD, Borowitz MJ, Porwit A, et al. The 2008 revision of the World Health Organization (WHO) classification of myeloid neoplasms and acute leukemia: rationale and important changes. Blood 2009;114(5):937-51.

[8] Balduini CL, Guarnone R, Pecci A, Centenara E, Ascari E. International prognostic scoring system and other prognostic systems for myelodysplastic syndromes. Blood 1997;90(10):4232-4.

[9] Malcovati L, Germing U, Kuendgen A, la Porta MG, Pascutto C, Invernizzi $\mathrm{R}$, et al. Time-dependent prognostic scoring system for predicting survival and leukemic evolution in myelodysplastic syndromes. J Clin Oncol 2007;25(23):3503-10.

[10] Greenberg P, Cox C, LeBeau MM, Fenaux P, Morel P, Sanz G, et al. International scoring system for evaluating prognosis in myelodysplastic syndromes. Blood 1997;89(6):2079-88.

[11] Sekeres MA, Schoonen WM, Kantarjian H, List A, Fryzek J, Paquette R, et al. Characteristics of US patients with myelodysplastic syndromes: results of six cross-sectional physician surveys. J Natl Cancer Inst 2008;100(21): 1542-51.

[12] Miller KB, Kim K, Morrison FS, Winter JN, Bennett JM, Neiman RS, et al. The evaluation of low-dose cytarabine in the treatment of myelodysplastic syndromes: a phase-III intergroup study. Ann Hematol 1992;65(4):162-8.

[13] Kantarjian H, Issa JP, Rosenfeld CS, Bennett JM, Albitar M, DiPersio J, et al. Decitabine improves patient outcomes in myelodysplastic syndromes: results of a phase III randomized study. Cancer 2006;106(8):1794-803.

[14] Knipp S, Hildebrand B, Kundgen A, Giagounidis A, Kobbe G, Haas R, et al. Intensive chemotherapy is not recommended for patients aged $>60$ years who have myelodysplastic syndromes or acute myeloid leukemia with high-risk karyotypes. Cancer 2007;110(2):345-52.

[15] Quesnel B, Guillerm G, Vereecque R, Wattel E, Preudhomme C, Bauters F, et al. Methylation of the p15(INK4b) gene in myelodysplastic syndromes is frequent and acquired during disease progression. Blood 1998;91(8):2985-90.

[16] Jiang Y, Dunbar A, Gondek LP, Mohan S, Rataul M, O'Keefe C, et al. Aberrant DNA methylation is a dominant mechanism in MDS progression to AML. Blood 2009;113(6):1315-25.
[17] Santini V, Kantarjian HM, Issa JP. Changes in DNA methylation in neoplasia: pathophysiology and therapeutic implications. Ann Intern Med 2001;134(7):573-86.

[18] Santini V. Azacitidine: activity and efficacy as an epigenetic treatment of myelodysplastic syndromes. Expert Rev Hematol 2009;2(2):121-7.

[19] Stresemann C, Bokelmann I, Mahlknecht U, Lyko F. Azacytidine causes complex DNA methylation responses in myeloid leukemia. Mol Cancer Ther 2008;7(9):2998-3005.

[20] Fenaux P, Mufti GJ, Hellström-Lindberg E, Santini V, Finelli C, Giagounidis A, et al. Efficacy of azacitidine compared with that of conventional care regimens in the treatment of higher-risk myelodysplastic syndromes: a randomised, openlabel, phase III study. Lancet Oncol 2009;10(3):223-32.

[21] Celgene Europe Ltd. Vidaza ${ }^{\circledR}$ Summary of product characteristics; 2008.

[22] Silverman LR, Demakos EP, Peterson BL, Kornblith AB, Holland JC, OdchimarReissig R, et al. Randomized controlled trial of azacitidine in patients with the myelodysplastic syndrome: a study of the cancer and leukemia group B. J Clin Oncol 2002;20(10):2429-40.

[23] Silverman LR, McKenzie DR, Peterson BL, Holland JF, Backstrom JT, Beach CL, et al. Further analysis of trials with azacitidine in patients with myelodysplastic syndrome: studies 8421,8921 , and 9221 by the Cancer and Leukemia Group B. J Clin Oncol 2006;24(24):3895-903.

[24] Silverman LR, McKenzie DR, Peterson BL, Demakos EP, Malone NT, Holland JF, et al. Azacitidine prolongs survival and time to AML transformation in highrisk myelodysplastic syndrome (MDS) patients $\geq 65$ years of age. Blood (ASH Annual Meeting Abstracts) 2005;106:2524.

[25] Kornblith AB, Herndon JE, Silverman LR, Demakos EP, Odchimar-Reissig R, Holland JF, et al. Impact of azacytidine on the quality of life of patients with myelodysplastic syndrome treated in a randomized phase III trial: a Cancer and Leukemia Group B study. J Clin Oncol 2002;20(10):2441-52.

[26] Seymour JF, Fenaux P, Silverman LR, Mufti SJ, Hellström-Lindberg E, Santini $\mathrm{V}$, et al. Effects of azacitidine compared with conventional care regimens in elderly ( $\geq 75$ years) patients with higher-risk myelodysplastic syndromes. Crit Rev Oncol Hematol 2010; in press.

[27] Fenaux P, Mufti GJ, Hellström-Lindberg E, Santini V, Gattermann N, Germin U, et al. Azacitidine prolongs overall survival compared with conventional care regimens in elderly patients with low bone marrow blast count acute myeloid leukemia. J Clin Oncol 2010;28(4):562-9.

[28] Lyons RM, Cosgriff TM, Modi SS, Gersh RH, Hainsworth JD, Cohn AL, et al. Hematologic response to three alternative dosing schedules of azacitidine in patients with myelodysplastic syndromes. J Clin Oncol 2009;27(11): 1850-6.

[29] Silverman LR, Holland JF, Weinberg RS, Alter BP, Davis RB, Ellison RR, et al. Effects of treatment with 5-azacytidine on the in vivo and in vitro hematopoiesis in patients with myelodysplastic syndromes. Leukemia 1993;7(Suppl. 1): 21-9.

[30] Sekeres MA, Maciejewski JP, Donley DW, Grinblatt DL, Narang M, Malone JM, et al. A study comparing dosing regimens and efficacy of subcutaneous to intravenous azacitidine (AZA) for the treatment of myelodysplastic syndromes (MDS). Blood (ASH Annual Meeting Abstracts) 2009;114:3797.

[31] Garcia-Manero G, Gore SD, Skikne B, Cogle CR, Ning Y, MacBeth KJ, et al. A Phase 1, Open-Label, Dose-Escalation Study to evaluate the safety, pharmacokinetics, and pharmacodynamics of oral azacitidine in patients with myelodysplastic syndromes (MDS) or acute myelogenous leukemia (AML). Blood (ASH Annual Meeting Abstracts) 2009;114(22):117.

[32] Silverman LR, Fenaux P, Mufti GJ, Santini V, Hellström-Lindberg E, Gattermann $\mathrm{N}$, et al. The effects of continued azacitidine (AZA) treatment cycles on response in high-risk patients (Pts) with myelodysplastic syndromes (MDS). Blood (ASH Annual Meeting Abstracts) 2008;112:227.

[33] List AF, Fenaux P, Mufti GJ, Hellström-Lindberg E, Gore S, Bennett JM, et al. Effect of azacitidine (AZA) on overall survival in higher-risk myelodysplastic syndromes (MDS) without complete remission. J Clin Oncol 2008;26(Suppl): 7006.

[34] Santini V, Fenaux P, Mufti G, Hellström-Lindberg E, List A, Silverman L, et al. Patient outcome measures during prolonged survival in patients with high-risk myelodysplastic syndromes (MDS) treated with azacitidine (AZA). J Clin Oncol 2008;26(Suppl):7028.

[35] Zwierzina H, Suciu S, Loeffler-Ragg J, Neuwirtova R, Fenaux P, Beksac M, et al. Low-dose cytosine arabinoside (LD-AraC) vs LD-AraC plus granulocyte/macrophage colony stimulating factor vs LD-AraC plus interleukin-3 for myelodysplastic syndrome patients with a high risk of developing acute leukemia: final results of a randomized phase III study (06903) of the EORTC Leukemia Cooperative Group. Leukemia 2005;19(11):1929-33.

[36] Santini V, Fenaux P, Mufti GJ, Hellström-Lindberg E, Silverman LB, List A, et al. Management and supportive care measures for adverse events in patients with myelodysplastic syndromes treated with azacitidine. Eur J Haematol; in press.

[37] Platzbecker U, Aul C, Ehninger G, Giagounidis A. Reduction of 5-azacitidine induced skin reactions in MDS patients with evening primrose oil. Ann Hematol 2010;89(4):427-8.

[38] Field T, Perkins J, Huang Y, Kharfan-Dabaja MA, Alsina M, Ayala E, et al. 5-Azacitidine for myelodysplasia before allogeneic hematopoietic cell transplantation. Bone Marrow Transplant 2010;45(2):255-60.

[39] Grövdal M, Khan R, Aggerholm A, Antunovic P, Astermark J, Bernell P, et al. Maintenance treatment with 5-azacitidine for patients with high risk myelodysplastic syndrome (MDS) or acute myeloid leukemia following MDS (MDS-AML) in complete remission (CR) after induction chemotherapy. Blood (ASH Annual Meeting Abstracts) 2008;112:223. 
[40] Gardin C, Prébet T, Bouabdallah K, Caillot D, Guerci A, Raffoux E, et al. A Phase II study of post-remission therapy with azacitidine (AZA) in patients with AML post-MDS and high-risk MDS: a GFM Group study. Blood (ASH Annual Meeting Abstracts) 2009:114:844.

[41] Sekeres MA, List AF, Cuthbertson D, Paquette R, Latham D, Afable M, et al. Phase I combination study of lenalidomide and azacitidine in patients with higher-risk myelodysplastic syndromes. J Clin Oncol 2010;28: 2253-8.
[42] Kantarjian H, Giles F, Greenberg P, Paquette R, Wang E, Gabrilove J, et al. Effect of romiplostim in patients (pts) with low or intermediate risk myelodysplastic syndrome (MDS) receiving azacytidine. Blood (ASH Annual Meeting Abstracts) 2008;112:224.

[43] Musto P, Maurillo L, Spagnoli A, Gozzini A, Rivellini F, Lunghi M, et al. Azacitidine for the treatment of lower risk myelodysplastic syndromes: a retrospective study of 74 patients enrolled in an Italian named patient program. Cancer 2010;116:1485-94. 